\title{
MARCINKIEWICZ SPACES, GARSIA-RODEMICH SPACES AND THE SCALE OF JOHN-NIRENBERG SELF IMPROVING INEQUALITIES
}

\author{
Mario Milman \\ CONICET, Instituto Argentino de Matemática \\ Saavedra 15, 1083 Buenos Aires, Argentina; mario.milman@gmail.com
}

\begin{abstract}
We extend to $n$-dimensions a characterization of the Marcinkiewicz $L(p, \infty)$ spaces first obtained by Garsia-Rodemich in the one dimensional case. This leads to a new proof of the John-Nirenberg self-improving inequalities. We also show a related result that provides still a new characterization of the $L(p, \infty)$ spaces in terms of distribution functions, reflects the self-improving inequalities directly, and also characterizes $L(\infty, \infty)$, the rearrangement invariant hull of $B M O$. We show an application to the study of tensor products with $L(\infty, \infty)$ spaces, which complements the classical work of O'Neil [19] and the more recent work of Astashkin [2].
\end{abstract}

\section{Introduction}

In their seminal paper [11], John-Nirenberg introduced the space $B M O$ and proved the celebrated John-Nirenberg inequality for functions in BMO. It is also well known, although perhaps somewhat less so, that in the same paper, JohnNirenberg showed that the $B M O$ self improvement inequality can be refined and framed as a scale of inequalities. These inequalities (or embeddings) are associated with what we nowadays call "John-Nirenberg spaces". This result of John-Nirenberg, which we now describe, is the starting point of our development in this paper.

Let $Q_{0} \subset \mathbf{R}^{n}$, be a fixed cube ${ }^{1}, 1 \leq p<\infty$. Let

$$
\begin{aligned}
P\left(Q_{0}\right)=\left\{\left\{Q_{i}\right\}_{i \in N}:\right. & \text { countable families of subcubes } Q_{i} \subset Q_{0}, \\
& \text { with pairwise disjoint interiors }\} .
\end{aligned}
$$

The John-Nirenberg spaces are defined by

$$
J N_{p}\left(Q_{0}\right)=\left\{f \in L^{1}\left(Q_{0}\right): J N_{p}\left(f, Q_{0}\right)<\infty\right\},
$$

where $^{2}$

$$
J N_{p}\left(f, Q_{0}\right)=\sup _{\left\{Q_{i}\right\}_{i} \in P\left(Q_{0}\right)}\left\{\left\{\sum_{i}\left|Q_{i}\right|\left(\frac{1}{\left|Q_{i}\right|} \int_{Q_{i}}\left|f-f_{Q_{i}}\right| d x\right)^{p}\right\}^{1 / p}\right\} .
$$

doi:10.5186/aasfm.2016.4129

2010 Mathematics Subject Classification: Primary 42B35, 46E30.

Key words: John-Nirenberg inequality, rearrangement, $B M O$.

The author was partially supported by a grant from the Simons Foundation (\#207929 to Mario Milman).

${ }^{1} \mathrm{~A}$ "cube" in this paper will always mean a cube with sides parallel to the coordinate axes.

${ }^{2}$ In what follows, as usual, $f_{Q}=\frac{1}{|Q|} \int_{Q} f d x$. 
Let us also recall that, for a given measure space, the Marcinkiewicz $L(p, \infty)$ spaces, $1 \leq p<\infty$, are defined by demanding ${ }^{3}$ that $\|f\|_{L(p, \infty)}^{*}<\infty$, where

$$
\|f\|_{L(p, \infty)}^{*}=\sup _{t>0}\left\{f^{*}(t) t^{1 / p}\right\}=\sup _{t>0}\left\{t\left(\lambda_{f}(t)\right)^{1 / p}\right\}
$$

while for $p=\infty$, the space $L(\infty, \infty)$ (cf. [3]) is defined ${ }^{4}$ by the condition $\|f\|_{L(\infty, \infty)}<$ $\infty$, where

$$
\|f\|_{L(\infty, \infty)}=\sup _{t>0}\left\{f^{* *}(t)-f^{*}(t)\right\}
$$

and

$$
f^{* *}(t)=\frac{1}{t} \int_{0}^{t} f^{*}(s) d s
$$

Then (cf. [11, Lemma 3], and also [21, Theorem 4.1, p. 209] for a more detailed proof).

Theorem 1. Let $1<p<\infty$. Suppose that $f \in J N_{p}\left(Q_{0}\right)$, then $f-f_{Q_{0}} \in$ $L(p, \infty)\left(Q_{0}\right)$, and there exists a constant $A\left(p, Q_{0}, n\right)$ such that

$$
\left\|f-f_{Q_{0}}\right\|_{L(p, \infty)\left(Q_{0}\right)} \leq A\left(p, Q_{0}, n\right) J N_{p}\left(f, Q_{0}\right)
$$

In particular,

$$
f-f_{Q_{0}} \in \bigcap_{r<p} L^{r}\left(Q_{0}\right)
$$

The limiting condition defining $J N_{p}\left(Q_{0}\right)$ when $p=\infty$ corresponds $^{5}$ to $B M O$, and in this case Theorem 1 corresponds to a version of the well known John-Nirenberg inequality [11].

In the one dimensional case, Garsia and Rodemich [10] improved on Theorem 1. To formulate the Garsia and Rodemich result it will be convenient to introduce a different scale of spaces which we shall term Garsia-Rodemich spaces. It will be useful for later use to give the relevant definitions in the $n$-dimensional case.

Let $Q_{0} \subset \mathbf{R}^{n}$ be a fixed cube, let $1 \leq p<\infty$, and let $p^{\prime}$ be defined by $\frac{1}{p}+\frac{1}{p^{\prime}}=1$. The Garsia-Rodemich spaces $\operatorname{GaRo}_{p}\left(Q_{0}\right)$ are defined as follows. We shall say that $f \in \operatorname{GaRo}_{p}\left(Q_{0}\right)$, if and only if $f \in L^{1}\left(Q_{0}\right)$, and $\exists C>0$ such that for all $\left\{Q_{i}\right\}_{i \in N}$ $\in P\left(Q_{0}\right)$ we have

$$
\sum_{i} \frac{1}{\left|Q_{i}\right|} \int_{Q_{i}} \int_{Q_{i}}|f(x)-f(y)| d x d y \leq C\left(\sum_{i}\left|Q_{i}\right|\right)^{1 / p^{\prime}}
$$

We let

$$
\operatorname{GaRo}_{p}\left(f, Q_{0}\right)=\inf \{C>0: \text { such that }(1.2) \text { holds }\} .
$$

Then we have (cf. [10])

\footnotetext{
${ }^{3}$ Here $f^{*}$ denotes the non-increasing rearrangement of $f$ and $\lambda_{f}$ its distribution function (cf. [4]).

${ }^{4}$ Some authors (including sometimes the author of this paper) use a different notation and let $W$ denote what we call $L(\infty, \infty)$. at the same time that they use the notation $L(\infty, \infty)=L^{\infty}$.

${ }^{5}$ The $J N_{\infty}\left(Q_{0}\right)$ condition would read

$$
\sup _{\left\{Q_{i}\right\}_{i} \in P\left(Q_{0}\right)} \frac{1}{\left|Q_{i}\right|} \int_{Q_{i}}\left|f-f_{Q_{i}}\right| d x<\infty .
$$
}


Theorem 2. ${ }^{6}$ Let $1<p<\infty$, and let $Q_{0}=I=[0,1]$. Then, as sets

$$
\operatorname{GaRo}_{p}(I)=L(p, \infty)(I) .
$$

Remark 1. The elementary proof of the embedding $L(p, \infty) \subset \mathrm{GaRo}_{p}$ outlined in [10] works in $n$-dimensions and actually shows that (cf. Theorem 5 part (ii), below)

$$
\operatorname{GaRo}_{p}(f, I) \leq \frac{p}{p-1} 2\|f\|_{L(p, \infty)(I)}^{*} .
$$

By Theorem ${ }^{7} 1$ we have

$$
J N_{p}(I) \subsetneq L(p, \infty)(I)
$$

therefore by Theorem 2 (cf. Section 2 below for a direct proof of the $n$ dimensional case) it follows that

$$
J N_{p}(I) \subsetneq \operatorname{GaRo}_{p}(I) .
$$

In conclusion, Theorem 2 not only improves on Theorem 1 in the one dimensional case, but also gives us an interesting characterization of the Marcinkiewicz $L(p, \infty)(I)$ spaces, $1<p<\infty$. Unfortunately, one part of the proof of Theorem 2 uses a nontrivial rearrangement inequality, also due to Garsia-Rodemich [10], which is only proved there in the one dimensional ${ }^{8}$ case.

In [10], the authors briefly suggest a possible different method to prove Theorem 2 in $n$-dimensions, and without dimensional constants, but no details are provided ${ }^{9}$. In this note we give a new proof Theorem 2 that is valid in $n$ dimensions (cf. Theorem 5 below) . Our approach is different from the one given in [10], and does not use martingale techniques. Instead, our method is ultimately based on Calderón-Zygmund type decompositions, following classical ideas ${ }^{10}$ in [3].

As we shall see (cf. Section 2) the verification that the John-Nirenberg conditions are stronger than the Garsia-Rodemich conditions (e.g. (1.4)) is immediate. Therefore, the crucial aspect of this approach to the John-Nirenberg theorem is the fact that the Garsia-Rodemich spaces are the same as the Marcinkiewicz $L(p, \infty)$ spaces! This clarifies the self improvement results of John-Nirenberg. Moreover, these ideas could potentially be useful in the investigation of related issues, e.g. the dimensional constants involved in the John-Nirenberg embeddings (cf. [7]).

Now the classical definitions of the $L(p, \infty)$ spaces are given in terms of growth conditions on rearrangements or distribution functions (cf. [4], [5], [18], [6], [20], etc.). The case $p=\infty$, which corresponds to $L(\infty, \infty)$ ("the rearrangement invariant hull of $B M O$ ", cf. [3]), also admits a similar characterization through the use of the oscillation operator $f^{* *}-f^{*}$, and indeed one can find a characterization of all the

\footnotetext{
${ }^{6}$ Here it seems appropriate to bring up the following. In his paper [8], Dyson writes "Professor Littlewood, when he makes use of an algebraic identity always saves himself the trouble of proving it; he maintains that an identity, if true, can be verified in a few lines by anybody obtuse enough to feel the need of verification. My object in the following pages is to confute this assertion". It is left to reader to decide if the author of the present paper is demonstrating his own obtuseness.

${ }^{7}$ The fact that the containment is strict was shown in [1].

${ }^{8}$ See also [12] for related inequalities.

${ }^{9}$ From [10, p. 115]: "We wish to point out also that using the Martingale techniques of [9] a proof of Theorem 2 can be obtained quite directly and without dimensional constants." We hope to follow up this suggestion elsewhere.

${ }^{10}$ It has the drawback of containing constants that depend on the dimension.
} 
$L(p, \infty)$ spaces, $p \in(1, \infty]$, in the same fashion, namely

$$
\|f\|_{L(p, \infty)}^{\#}=\sup _{s}\left\{\left(f^{* *}(s)-f^{*}(s)\right) s^{1 / p}\right\}<\infty .
$$

This characterization, while extremely useful in many problems (cf. [4], [17]) is not always easy to implement, and does not reflect immediately the self improvement ${ }^{11}$ of the Garsia-Rodemich construction. In this direction, we found a different characterization of $L(\infty, \infty)$, which gives an implicit differential inequality reflecting the exponential decay of the distribution function of elements of $L(\infty, \infty)$, via the use of distribution functions (cf. Section 3 below)

Theorem 3. Let $(\Omega, \mu)$ be a measure space ${ }^{12}$. Then, $f \in L(\infty, \infty):=L(\infty, \infty)(\Omega)$ if and only if there exists $C>0$ such that for all $t>0$,

$$
\int_{t}^{\infty} \lambda_{f}(s) d s \leq C \lambda_{f}(t)
$$

and

$$
\|f\|_{L(\infty, \infty)}^{\# \#}:=\inf \{C: \text { such that (1.5) holds }\}=\|f\|_{L(\infty, \infty)} .
$$

This characterization gives immediately the exponential integrability of functions in $L(\infty, \infty)$, via the implicit differential inequality (1.5). In fact, it is also welcome that there is a similar characterization for all $L(p, \infty)$ spaces, $1<p<\infty$.

Theorem 4. Let $1<p<\infty$. Let $(\Omega, \mu)$ be a measure space. Then,

$$
\begin{aligned}
L(p, \infty) & :=L(p, \infty)(\Omega)=\left\{f \in L_{\mathrm{loc}}^{1}(\Omega):\|f\|_{L(p, \infty)}^{*}=\sup _{s}\left\{f^{*}(s) s^{1 / p}\right\}<\infty\right\} \\
& =\left\{f \in L_{\mathrm{loc}}^{1}(\Omega):\|f\|_{L(p, \infty)}=\sup _{s}\left\{f^{* *}(s) s^{1 / p}\right\}<\infty\right\},
\end{aligned}
$$

coincides with the set of all $f$ such that $f^{* *}(\infty)=0$, and

$$
\|f\|_{L(p, \infty)}^{\#}=\sup _{s>0}\left\{\left(f^{* *}(s)-f^{*}(s)\right) s^{1 / p}\right\}<\infty,
$$

which in turn coincides with the set of all $f$ such that $f^{* *}(\infty)=0$, and

$$
\|f\|_{L(p, \infty)}^{\# \#}=\sup _{t>0}\left\{\frac{1}{\left(\lambda_{f}(t)\right)^{1-1 / p}} \int_{t}^{\infty} \lambda_{f}(s) d s\right\}<\infty .
$$

If one combines (1.7) with the usual definition of the spaces $L(p, \infty)$ (cf. (1.6)), one readily obtains a known characterization of the $L(p, \infty)$ spaces which was apparently first given by O'Neil [19].

Corollary 1. Let $1<p<\infty$, then

$$
\|f\|_{L(p, \infty)}^{*} \sim \inf \left\{C^{1 / p}: \int_{t}^{\infty} \lambda_{f}(s) d s \leq C t^{1-p}\right\} .
$$

Remark 2. One difference between (1.7) and (1.8) is given by the fact that the former also works in the case $p=\infty$. Both formulations can be extended to more general Marcinkiewicz spaces, $M_{\phi}$, where $\phi$ is a concave function. In particular, we refer to [19] for the corresponding theory of generalized Marcinkiewicz spaces $M_{\phi}$ defined via (1.8).

\footnotetext{
${ }^{11}$ Note however that $\left(f^{* *}(t)-f^{*}(t)\right)=t \frac{d}{d t}\left(-f^{* *}(t)\right)$.

${ }^{12}$ When dealing with infinite measure spaces we shall always assume that all functions $f$ considered are such that their distribution functions $\lambda_{f}(t)$ are not equal to $\infty$, for all $t>0$.
} 
In his expansive work [19], O'Neil used the formulae (1.8 ) to study tensor products of $L(p, q)$ spaces (cf. also [2] and [13]). The space $L(\infty, \infty)$ was introduced later (cf. [3]), and consequently was not considered in [19]. In the last section of this paper we give an application of (1.5) to show that (cf. Theorem 6 in Section 4 below)

$$
L(\infty, \infty)\left(\Omega_{1}\right) \otimes L^{\infty}\left(\Omega_{2}\right) \subset L(\infty, \infty)\left(\Omega_{1} \times \Omega_{2}\right) .
$$

While we think that (1.9) could be useful in establishing other embeddings of tensor products involving $L(\infty, \infty)$, such an undertaking falls outside the scope of this note.

In conclusion, we should mention that this paper is part of series of papers by the author on $B M O$, self improvement and interpolation, that go back at least to [14], [15], [16], with the most recent opus being [17], to which we refer for background information and further references.

\section{John-Nirenberg spaces and Garsia-Rodemich spaces}

It is easy to see the connection of the John-Nirenberg spaces with $B M O$. Fix a cube $Q_{0} \subset \mathbf{R}^{n}$ and let

$$
\|f\|_{B M O\left(Q_{0}\right)}=\sup \left\{\frac{1}{|Q|} \int_{Q}\left|f-f_{Q}\right| d x: Q \text { subcube of } Q_{0}\right\} .
$$

Then, for $1 \leq p<\infty$,

$$
J N_{p}\left(f, Q_{0}\right) \leq\|f\|_{B M O\left(Q_{0}\right)}\left|Q_{0}\right|^{1 / p} .
$$

Indeed, if $\left\{Q_{i}\right\}_{i \in N} \in P\left(Q_{0}\right)$, then we clearly have

$$
\begin{aligned}
\left\{\sum_{i}\left|Q_{i}\right|\left(\frac{1}{\left|Q_{i}\right|} \int_{Q_{i}}\left|f-f_{Q_{i}}\right| d x\right)^{p}\right\}^{1 / p} & \leq\left\{\sum_{i}\left|Q_{i}\right|\left(\|f\|_{B M O\left(Q_{0}\right)}\right)^{p}\right\}^{1 / p} \\
& \leq\|f\|_{B M O\left(Q_{0}\right)}\left|Q_{0}\right|^{1 / p} .
\end{aligned}
$$

The purpose of this section is to prove the following

Theorem 5. Let $1<p<\infty$, and let $Q_{0} \subset R^{n}$ be a fixed cube. Then

(i) $J N_{p}\left(Q_{0}\right) \subset \operatorname{GaRo}_{p}\left(Q_{0}\right)$, in fact

$$
\operatorname{GaRo}_{p}\left(f, Q_{0}\right) \leq 2 J N_{p}\left(f, Q_{0}\right) .
$$

(ii) $\operatorname{GaRo}_{p}\left(Q_{0}\right)=L(p, \infty)\left(Q_{0}\right)$, in fact we have

$$
\begin{aligned}
\operatorname{GaRo}_{p}\left(f, Q_{0}\right) & \leq \frac{2 p}{p-1}\|f\|_{L(p, \infty)}^{*} \text { and } \\
\sup _{t} t^{1 / p}\left(f^{* *}(t)-f^{*}(t)\right) & \leq 2^{n / p^{\prime}+1} \operatorname{GaRo}_{p}\left(f, Q_{0}\right)+\left(\frac{4}{\left|Q_{0}\right|}\right)^{1 / p^{\prime}}\|f\|_{L^{1}} .
\end{aligned}
$$

Proof. (i) Suppose that $\left\{Q_{i}\right\}_{i \in N} \in P\left(Q_{0}\right)$. Then for all $Q_{i}, i \in N$, we have,

$$
\begin{aligned}
\int_{Q_{i}} \int_{Q_{i}}|f(x)-f(y)| d x d y & \leq \int_{Q_{i}} \int_{Q_{i}}\left|f(x)-f_{Q_{i}}\right| d x d y+\int_{Q_{i}} \int_{Q_{i}}\left|f_{Q_{i}}-f(y)\right| d x d y \\
& =2\left|Q_{i}\right| \int_{Q_{i}}\left|f-f_{Q_{i}}\right| d x .
\end{aligned}
$$


Therefore,

$$
\begin{aligned}
& \sum_{i} \frac{1}{\left|Q_{i}\right|} \int_{Q_{i}} \int_{Q_{i}}|f(x)-f(y)| d x d y \leq 2 \sum_{i} \int_{Q_{i}}\left|f-f_{Q_{i}}\right| d x \\
& =2 \sum_{i}\left|Q_{i}\right|^{1 / p^{\prime}}\left|Q_{i}\right|^{1 / p} \frac{1}{\left|Q_{i}\right|} \int_{Q_{i}}\left|f-f_{Q_{i}}\right| d x \\
& \leq 2\left(\sum_{i}\left|Q_{i}\right|\right)^{1 / p^{\prime}}\left\{\sum_{i}\left|Q_{i}\right|\left(\frac{1}{\left|Q_{i}\right|} \int_{Q_{i}}\left|f-f_{Q_{i}}\right| d x\right)^{p}\right\}^{1 / p}
\end{aligned}
$$

and (2.1) follows.

(ii) We show first that $L(p, \infty)\left(Q_{0}\right) \subset \operatorname{GaRo}_{p}\left(Q_{0}\right)$. Let $\left\{Q_{i}\right\}_{i \in N} \in P\left(Q_{0}\right)$, then

$$
\begin{aligned}
\sum_{i} \frac{1}{\left|Q_{i}\right|} \int_{Q_{i}} \int_{Q_{i}}|f(x)-f(y)| d x d y & \leq \sum_{i} \frac{1}{\left|Q_{i}\right|} \int_{Q_{i}} \int_{Q_{i}}(|f(x)|+|f(y)|) d x d y \\
& \leq 2 \int_{\cup Q_{i}}|f(x)| d x \leq 2 \int_{0}^{\sum_{i}\left|Q_{i}\right|} f^{*}(t) d t \\
& \leq 2\|f\|_{L(p, \infty)}^{*} \int_{0}^{\sum_{i}\left|Q_{i}\right|} t^{-1 / p} d t \\
& =\frac{2 p}{p-1}\|f\|_{L(p, \infty)}^{*}\left(\sum_{i}\left|Q_{i}\right|\right)^{1 / p^{\prime}} .
\end{aligned}
$$

Consequently,

$$
\operatorname{GaRo}_{p}\left(f, Q_{0}\right) \leq \frac{2 p}{p-1}\|f\|_{L(p, \infty)}^{*}
$$

To show the remaining inclusion, $\operatorname{GaRo}_{p}\left(Q_{0}\right) \subset L(p, \infty)\left(Q_{0}\right)$, we argue as in $[4$, Chapter 5]. We provide all the details for the sake of completeness.

To show that a function $f$ belongs to $L(p, \infty)\left(Q_{0}\right)$ it is equivalent to show that $|f| \in L(p, \infty)\left(Q_{0}\right)$, therefore, since

$$
\operatorname{GaRo}_{p}\left(|f|, Q_{0}\right) \leq \operatorname{GaRo}_{p}\left(f, Q_{0}\right)
$$

to show that $f \in \operatorname{GaRo}_{p}\left(Q_{0}\right)$ belongs to $L(p, \infty)\left(Q_{0}\right)$, we can assume without loss that $f \geq 0$. Let $f \in \operatorname{GaRo}_{p}\left(Q_{0}\right), f \geq 0$. Fix $t>0$, such that $t<\left|Q_{0}\right| / 4$, and let $E=\left\{x \in Q_{0}: f(x)>f^{*}(t)\right\}$. By definition, $|E| \leq t<\left|Q_{0}\right| / 4$, consequently, we can find a relatively open subset of $Q_{0}, \Omega$, say, such that $E \subset \Omega$ and $|\Omega| \leq 2 t \leq\left|Q_{0}\right| / 2$. By [4, Lemma 7.2, p. 377] we can find a sequence of cubes $\left\{Q_{i}\right\}_{i \in N}$, with pairwise disjoint interiors, such that

$$
\begin{aligned}
& \text { (i) }\left|\Omega \cap Q_{i}\right| \leq \frac{1}{2}\left|Q_{i}\right| \leq\left|\Omega^{c} \cap Q_{i}\right|, \quad i=1,2 \ldots, \\
& \text { (ii) } \Omega \subset \bigcup_{i \in N} Q_{i} \subset Q_{0}, \\
& \text { (iii) }|\Omega| \leq \sum_{i \in N}\left|Q_{i}\right| \leq 2^{n+1}|\Omega| .
\end{aligned}
$$


Now, to estimate $t^{1 / p}\left(f^{* *}(t)-f^{*}(t)\right)$, it will be more convenient, by homogeneity, to consider $t\left(f^{* *}(t)-f^{*}(t)\right)$ first. Then, we have

$$
\begin{aligned}
t\left(f^{* *}(t)-f^{*}(t)\right) & =\int_{E}\left\{f(x)-f^{*}(t)\right\} d x \leq \sum_{i \in N} \int_{E \cap Q_{i}}\left\{f(x)-f^{*}(t)\right\} d x \\
& =\sum_{i \in N}\left(\int_{E \cap Q_{i}}\left\{f(x)-f_{Q_{i}}\right\} d x+\left|E \cap Q_{i}\right|\left\{f_{Q_{i}}-f^{*}(t)\right\}\right) \\
& \leq \sum_{i \in N}\left(\int_{Q_{i}}\left\{f(x)-f_{Q_{i}}\right\} d x+\left|E \cap Q_{i}\right|\left\{f_{Q_{i}}-f^{*}(t)\right\}\right) \\
& =(I)+(I I) .
\end{aligned}
$$

Let $J=\left\{i: f_{Q_{i}}>f^{*}(t)\right\}$, then

$$
\begin{aligned}
(I I) & =\sum_{i \in N}\left|E \cap Q_{i}\right|\left\{f_{Q_{i}}-f^{*}(t)\right\} \leq \sum_{i \in J}\left|E \cap Q_{i}\right|\left\{f_{Q_{i}}-f^{*}(t)\right\} \\
& \leq \sum_{i \in J}\left|\Omega \cap Q_{i}\right|\left\{f_{Q_{i}}-f^{*}(t)\right\} \leq \sum_{i \in J}\left|\Omega^{c} \cap Q_{i}\right|\left\{f_{Q_{i}}-f^{*}(t)\right\} \\
& \left.=\sum_{i \in J} \int_{\Omega^{c} \cap Q_{i}}\left\{f_{Q_{i}}-f^{*}(t)\right\} d x \leq \sum_{i \in J} \int_{\Omega^{c} \cap Q_{i}}\left\{f_{Q_{i}}-f(x)\right\} d x \quad \text { since } \Omega^{c} \subset E^{c}\right) \\
& \leq \sum_{i \in J} \int_{Q_{i}}\left|f_{Q_{i}}-f(x)\right| d x \leq \sum_{i \in J} \frac{1}{\left|Q_{i}\right|} \int_{Q_{i}} \int_{Q_{i}}|f(y)-f(x)| d x d y \\
& \leq \operatorname{GaRo}_{p}\left(f, Q_{0}\right)\left(\sum_{i \in N}\left|Q_{i}\right|\right)^{1 / p^{\prime}}
\end{aligned}
$$

Likewise,

$$
\begin{aligned}
(I) & =\sum_{i \in N} \int_{Q_{i}}\left\{f(x)-f_{Q_{i}}\right\} d x=\sum_{i \in N} \frac{1}{\left|Q_{i}\right|} \int_{Q_{i}} \int_{Q_{i}}(f(x)-f(y)) d x d y \\
& \leq \sum_{i \in N} \frac{1}{\left|Q_{i}\right|} \int_{Q_{i}} \int_{Q_{i}}|f(x)-f(y)| d x d y \leq \operatorname{GaRo}_{p}\left(f, Q_{0}\right)\left(\sum_{i \in N}\left|Q_{i}\right|\right)^{1 / p^{\prime}} .
\end{aligned}
$$

Combining the inequalities we have obtained,

$$
\begin{aligned}
t\left(f^{* *}(t)-f^{*}(t)\right) & \leq 2 \operatorname{GaRo}_{p}\left(f, Q_{0}\right)\left(\sum_{i \in N}\left|Q_{i}\right|\right)^{1 / p^{\prime}} \\
& \leq 2 \operatorname{GaRo}_{p}\left(f, Q_{0}\right)\left(2^{n+1}\right)^{1 / p^{\prime}} 2^{-1 / p^{\prime}} t^{1 / p^{\prime}}
\end{aligned}
$$

Therefore,

$$
\sup _{t \leq\left|Q_{0}\right| / 4} t^{1 / p}\left(f^{* *}(t)-f^{*}(t)\right) \leq 2^{n / p^{\prime}+1} \operatorname{GaRo}_{p}\left(f, Q_{0}\right) .
$$

To deal with $t>\left|Q_{0}\right| / 4$, we note that

$$
t\left(f^{* *}(t)-f^{*}(t)\right)=\int_{f^{*}(t)}^{\infty} \lambda_{f}(s) d s \leq \int_{0}^{\infty} \lambda_{f}(s) d s=\|f\|_{L^{1}}
$$


therefore,

$$
t^{1 / p}\left(f^{* *}(t)-f^{*}(t)\right) \leq t^{-1 / p^{\prime}}\|f\|_{L^{1}} \leq\left(\frac{4}{\left|Q_{0}\right|}\right)^{1 / p^{\prime}}\|f\|_{L^{1}}
$$

Thus,

$$
\sup _{t} t^{1 / p}\left(f^{* *}(t)-f^{*}(t)\right) \leq 2^{n / p^{\prime}+1} \operatorname{GaRo}_{p}\left(f, Q_{0}\right)+\left(\frac{4}{\left|Q_{0}\right|}\right)^{1 / p^{\prime}}\|f\|_{L^{1}},
$$

and the desired result follows by Theorem 4 .

\section{Another characterization of the $L(p, \infty)$ spaces, $1<p \leq \infty$}

The purpose of this section is to give a proof of Theorem 3 and Theorem 4 . We start with the former.

Proof. Let $f$ be such that there exists $C>0$ such that (1.5) holds for all $t>0$. Then, we have

$$
\left(f^{* *}(t)-f^{*}(t)\right) t=\int_{f^{*}(t)}^{\infty} \lambda_{f}(s) d s \leq C \lambda_{f}\left(f^{*}(t)\right) \leq C t .
$$

Thus,

$$
\|f\|_{L(\infty, \infty)} \leq \inf \{C:(1.5) \text { holds }\}=\|f\|_{L(\infty, \infty)}^{\# \#} .
$$

Conversely, suppose that $f \in L(\infty, \infty)$. Then, for all $t>0$, we have,

$$
\int_{f^{*}(t)}^{\infty} \lambda_{f}(s) d s=\left(f^{* *}(t)-f^{*}(t)\right) t \leq t\|f\|_{L(\infty, \infty)} .
$$

Therefore,

$$
\int_{f^{*}\left(\lambda_{f}(t)\right)}^{\infty} \lambda_{f}(s) d s \leq \lambda_{f}(t)\|f\|_{L(\infty, \infty)} .
$$

Now, since $f^{*}\left(\lambda_{f}(t)\right) \leq t$, we have

$$
\int_{t}^{\infty} \lambda_{f}(s) d s \leq \lambda_{f}(t)\|f\|_{L(\infty, \infty)}
$$

Consequently,

concluding the proof.

$$
\|f\|_{L(\infty, \infty)}^{\# \#} \leq\|f\|_{L(\infty, \infty)}
$$

We proceed with the proof of Theorem 4 .

Proof. We trivially have $\|f\|_{L(p, \infty)}^{\#} \leq\|f\|_{L(p, \infty)}$. Moreover, if $f^{* *}(\infty)=0$, then

$$
\begin{aligned}
f^{* *}(t) t^{1 / p} & =t^{1 / p} \int_{t}^{\infty}\left(f^{* *}(s)-f^{*}(s)\right) \frac{d s}{s}=t^{1 / p} \int_{t}^{\infty}\left(f^{* *}(s)-f^{*}(s)\right) s^{1 / p} s^{-1 / p} \frac{d s}{s} \\
& \leq t^{1 / p}\|f\|_{L(p, \infty)}^{\#} \int_{t}^{\infty} s^{-1 / p} \frac{d s}{s}=p\|f\|_{L(p, \infty)}^{\#} .
\end{aligned}
$$

Consequently,

$$
\|f\|_{L(p, \infty)} \leq p\|f\|_{L(p, \infty)}^{\#} .
$$

The last part of the result follows exactly as the proof of Theorem 3 (the case $p=\infty$ ). For example, from

$$
\int_{t}^{\infty} \lambda_{f}(s) d s \leq\|f\|_{L(p, \infty)}^{\# \#}\left(\lambda_{f}(t)\right)^{1-1 / p}
$$


we get

and therefore

$$
\left(f^{* *}(t)-f^{*}(t)\right) t=\int_{f^{*}(t)}^{\infty} \lambda_{f}(s) d s \leq\|f\|_{L(p, \infty)}^{\# \#} t^{1-1 / p}
$$

Conversely, for all $t>0$,

$$
\|f\|_{L(p, \infty)}^{\#} \leq\|f\|_{L(p, \infty)}^{\# \#}
$$

$$
t\|f\|_{L(p, \infty)}^{\#} \geq t t^{1 / p}\left(f^{* *}(t)-f^{*}(t)\right) \geq t^{1 / p} \int_{f^{*}(t)}^{\infty} \lambda_{f}(s) d s
$$

Thus,

$$
\lambda_{f}(t)\|f\|_{L(p, \infty)}^{\#} \geq\left(\lambda_{f}(t)\right)^{1 / p} \int_{f^{*}\left(\lambda_{f}(t)\right)}^{\infty} \lambda_{f}(s) d s \geq\left(\lambda_{f}(t)\right)^{1 / p} \int_{t}^{\infty} \lambda_{f}(s) d s,
$$

and the desired result follows.

Remark 3. Observe that when $p=1$, the previous considerations provide a characterization of $L^{1}$, not of $L(1, \infty)$. Indeed, the corresponding result for $p=1$ is

$$
\sup _{t} f^{* *}(t) t=\sup _{t} \int_{0}^{t} f^{*}(s) d s=\|f\|_{L^{1}}=\sup _{t} \int_{t}^{\infty} \lambda_{f}(s) d s
$$

In other words, $L^{1}$ is characterized by the condition

$$
\sup _{t>0} \int_{t}^{\infty} \lambda_{f}(s) d s \leq C .
$$

Note that in this case $\left(\lambda_{f}(t)\right)^{1-1 / 1}=1$.

To conclude this section we prove Corollary 1.

Lemma 1. Let $1<p<\infty$. Then

$$
\|f\|_{L(p, \infty)}^{\# \#} \approx \inf \left\{C^{1 / p}: \int_{t}^{\infty} \lambda_{f}(s) d s \leq C t^{1-p}\right\} .
$$

Proof. Note that

$$
\left(\lambda_{f}(t)\right)^{1 / p} \leq\|f\|_{L(p, \infty)} t^{-1} \Leftrightarrow \lambda_{f}(t) \leq\|f\|_{L(p, \infty)}^{p} t^{-p} .
$$

Suppose that $f \in L(p, \infty)$. By the previous Theorem,

$$
\begin{aligned}
\int_{t}^{\infty} \lambda_{f}(s) d s & \leq\|f\|_{L(p, \infty)}^{\# \#}\left(\lambda_{f}(t)\right)^{1-1 / p} \\
& \leq\|f\|_{L(p, \infty)}^{\# \#}\|f\|_{L(p, \infty)}^{p(1-1 / p)} t^{-p(1-1 / p)} \\
& \leq C\|f\|_{L(p, \infty)}^{p} t^{1-p} .
\end{aligned}
$$

Conversely, suppose that

$$
\int_{t}^{\infty} \lambda_{f}(s) d s \leq C t^{1-p}
$$

Then, since $\lambda_{f}$ decreases,

$$
\begin{aligned}
\lambda_{f}(t)^{1 / p} \frac{t}{2} & \leq \int_{t / 2}^{t} \lambda_{f}(s)^{1 / p} d s=\int_{t / 2}^{t} \lambda_{f}(s) \lambda_{f}(s)^{1 / p-1} d s \leq \lambda_{f}(t / 2)^{1 / p-1} \int_{t / 2}^{t} \lambda_{f}(s) d s \\
& \leq \lambda_{f}(t / 2)^{1 / p-1} \int_{t / 2}^{\infty} \lambda_{f}(s) d s \leq \lambda_{f}(t / 2)^{1 / p-1} C 2^{p-1} t^{1-p} .
\end{aligned}
$$


Therefore,

and, consequently,

$$
\lambda_{f}(t / 2)^{-1 / p+1} \lambda_{f}(t)^{1 / p} \leq \tilde{C} t^{-p}
$$

The desired result follows.

$$
\lambda_{f}(t)^{-1 / p+1} \lambda_{f}(t)^{1 / p} \leq \tilde{C} t^{-p}
$$

\section{Final remarks}

4.1. Tensor products with $L(\infty, \infty)$. The new formula we presented for the computation of the "norm" \|\|$_{L(\infty, \infty)}$ has several applications. Here, following O'Neil [19] (cf. also [2], [13]), we shall briefly consider tensor products with $L(\infty, \infty)$. It is not our purpose to develop the most general results, but to give a flavor of the ideas involved.

Theorem 6. Let $\left(\Omega_{1}, \mu_{1}\right),\left(\Omega_{2}, \mu_{2}\right)$, be measure spaces. Then,

$$
L(\infty, \infty)\left(\Omega_{1}\right) \otimes L^{\infty}\left(\Omega_{2}\right) \subset L(\infty, \infty)\left(\Omega_{1} \times \Omega_{2}\right),
$$

with

$$
\|f \otimes g\|_{L(\infty, \infty)\left(\Omega_{1} \times \Omega_{2}\right)} \leq\|f\|_{L(\infty, \infty)\left(\Omega_{1}\right)}\|g\|_{L^{\infty}\left(\Omega_{2}\right)} .
$$

Proof. Let $f \in L(\infty, \infty)\left(\Omega_{1}\right), g \in L^{\infty}\left(\Omega_{2}\right)$. The distribution function of $f \otimes g$ is computed in [19, Lemma 7.1 (2), p. 97]

$$
\lambda_{f \otimes g}(z)=\int_{0}^{\infty} \lambda_{f}\left(\frac{z}{u}\right) d\left(-\lambda_{g}(u)\right), \quad z>0 .
$$

Therefore, on account that $g \in L^{\infty}$, we have

$$
\lambda_{f \otimes g}(z)=\int_{0}^{\|g\|_{L^{\infty}\left(\Omega_{2}\right)}} \lambda_{f}\left(\frac{z}{u}\right) d\left(-\lambda_{g}(u)\right) .
$$

Then, by Tonnelli's theorem, for all $t>0$,

$$
\begin{aligned}
\int_{t}^{\infty} \lambda_{f \otimes g}(z) d z & =\int_{t}^{\infty} \int_{0}^{\|g\|_{L^{\infty}\left(\Omega_{2}\right)}} \lambda_{f}\left(\frac{z}{u}\right) d\left(-\lambda_{g}(u)\right) d z \\
& =\int_{0}^{\|g\|_{L^{\infty}\left(\Omega_{2}\right)}} \int_{t}^{\infty} \lambda_{f}\left(\frac{z}{u}\right) d z d\left(-\lambda_{g}(u)\right) \\
& =\int_{0}^{\|g\|_{L^{\infty}\left(\Omega_{2}\right)}} \int_{\frac{t}{u}}^{\infty} \lambda_{f}(r) u d r d\left(-\lambda_{g}(u)\right) \\
& \leq\|g\|_{L^{\infty}\left(\Omega_{2}\right)}\|f\|_{L(\infty, \infty)\left(\Omega_{1}\right)}^{\# \#} \int_{0}^{\|g\|_{L^{\infty}\left(\Omega_{2}\right)}} \lambda_{f}\left(\frac{t}{u}\right) d\left(-\lambda_{g}(u)\right) \\
& =\|g\|_{L^{\infty}\left(\Omega_{2}\right)}\|f\|_{L(\infty, \infty)\left(\Omega_{1}\right)}^{\# \#} \lambda_{f \otimes g}(t) \quad(\text { by }(4.3)) .
\end{aligned}
$$

Hence, by Theorem 3,

$$
\|f \otimes g\|_{L(\infty, \infty)\left(\Omega_{1} \times \Omega_{2}\right)}^{\# \#} \leq\|f\|_{L(\infty, \infty)\left(\Omega_{1}\right)}^{\# \# g \|_{L^{\infty}\left(\Omega_{2}\right)}} .
$$

4.2. More problems. 1. It seems to us that our approach to prove the second part of Theorem 5 can be modified to study the rearrangement inequality of GarsiaRodemich [10, Theorem 7.3] in the $n$-dimensional case.

2. It would be of interest to follow up on the suggestion of Garsia-Rodemich and prove a version of Theorem 5 using the methods of [9].

3. It would be of interest to complete the study of tensor products with $L(\infty, \infty)$. 
Acknowledgement. I am grateful to Professor Michael Cwikel for a number of suggestions that allowed me to clarify and improve the presentation of the paper.

\section{References}

[1] Aalto, D., L. Berkovits, O. E. Kansanen, and H. Yue: John-Nirenberg lemmas for a doubling measure. - Studia Math. 204, 2011, 21-37.

[2] Astashkin, S. V.: Tensor product in symmetric function spaces. - Collect. Math. 48, 1997, $375-391$.

[3] Bennett, C., R. DeVore, and R. Sharpley: Weak- $L^{\infty}$ and BMO. - Ann. of Math. (2) 113, 1981, 601-611.

[4] Bennett, C., and R. Sharpley: Interpolation of operators. - Academic Press, 1988.

[5] Calderón, A.P.: Spaces between $L^{1}$ and $L^{\infty}$ and the theorem of Marcinkiewicz. - Studia Math. 26, 1966, 273-299.

[6] Cwikel, M., and P. NiLsson: Interpolation of Marcinkiewicz spaces. - Math. Scand. 56, 1985, 29-42.

[7] Cwikel, M., Y. Sagher, and P. Shvartsman: A new look at the John-Nirenberg and John-Strömberg theorems for BMO. - J. Funct. Anal. 263, 2012, 129-167.

[8] Dyson, F. D.: Some guesses in the theory of partitions. - Eureka 8, 1944, 10-15.

[9] Garsia, A. M.: Martingale inequalities: Seminar notes on recent progress. - Mathematics Lecture Notes Series, W. A. Benjamin, Inc., Reading, Mass.-London-Amsterdam, 1973.

[10] Garsia, A. M., and E. Rodemich: Monotonicity of certain functional under rearrangements. - Ann. Inst. Fourier (Grenoble) 24, 1974, 67-116.

[11] John, F., and L. Nirenberg: On functions of bounded mean oscillation. - Comm. Pure Appl. Math. 14, 1961, 415-426.

[12] Martin, J., and M. Milman: Fractional Sobolev inequalities: symmetrization, isoperimetry and interpolation. - Astérisque 366, 2014.

[13] Milman, M.: Some new function spaces and their tensor products. - Bull. Aust. Math. Soc. 19, 1978, 147-149.

[14] Milman, M.: Rearrangements of $B M O$ functions and interpolation. - In: Lecture Notes in Math. 1070, 1984, 208-212.

[15] Milman, M.: A note on Gehring's lemma. - Ann. Acad. Sci. Fenn. Math. 21, 1996, 389-398.

[16] Milman, M.: A note on interpolation and higher integrability. - Ann. Acad. Sci. Fenn. Math. 23, 1998, 169-180.

[17] Milman, M.: BMO: Oscillations, self-improvement, Gagliardo coordinate spaces and reverse Hardy inequalities. - In: Harmonic Analysis, Partial Differential Equations, Banach Spaces, and Operator Theory. Celebrating Cora Sadosky's Life. Volume 1 (edited by S. Marcantognini, M. C. Pereyra, A. Stokolos and W. Urbina), AWM-Springer Series (to appear).

[18] OKLAnder, E. T.: Interpolación, espacios de Lorentz y teorema de Marcinkiewicz. - Cursos y Seminarios de Matemáticas 20, Univ. Buenos Aires, 1965.

[19] O'NeIL, R.: Integral transforms and tensor products on Orlicz spaces and $L(p, q)$ spaces. - J. Anal. Math. 21, 1968, 4-276.

[20] Pustylnik, E.: On some properties of generalized Marcinkiewicz spaces. - Studia Math. 144, 2001, 227-243.

[21] Torchinsky, A.: Real variable methods in harmonic analysis. - Academic Press, 1986. 